M.I.T Media Laboratory Perceptual Computing Section Technical Report No. 441

Appears: Proceedings of the IEEE Non-Rigid and Articulated Motion Workshop at CVPR'97, San Juan, June 16, 1997

\title{
A Three-Dimensional Model of Human Lip Motions Trained from Video
}

\author{
Sumit Basu and Alex Pentland \\ MIT Media Laboratory, 20 Ames St., Cambridge, MA 02139 USA \\ $\{$ sbasu,sandy\}@media.mit.edu
}

\begin{abstract}
We present a 3D model of human lips and develop a framework for training it from real data. The model starts off with generic physics specified with the finite element method and "learns" the correct physics through observations. The model's physics allow physically-based regularization between sparse observation points and the resulting set of deformations are used to derive the correct physical modes of the model. Preliminary results showing the model's ability to reconstruct lip shapes from sparse data are shown. The resulting model can be used for both analysis and synthesis.
\end{abstract}

\section{Introduction}

In the course of ordinary conversation and expression, the human lips can deform in a variety of interesting and non-rigid ways. Tracking these motions accurately has proved to be quite difficult. There are a number of reasons behind this, but primary among them is that all of the information about the lips is not observable at a given point. For example, we can at best see only the inner and outer contours of the lips from a given view. In addition, the presence of the tongue can obscure the inner contour, and the flesh tone/lighting can obscure the outer contour. we propose that with an accurate spatio-temporal model of the lips, this task will be greatly simplified. In essence, the argument is that a model that can only vary in the ways that the lips actually move will not be "fooled" by erroneous data. Such a model could form the heart of a robust estimation framework for finding the best estimate of the lip pose given whatever data was available. In addition, this model could be used to synthesize lip motions for computer graphics. In this study, we present a model and a means for training it that we feel will be able to provide these benefits for analysis and synthesis.

\subsection{Background}

In looking at the prior work, there are two major groups of lip models. The first of these contains the models developed for analysis, usually intended for input into a combined audiovisual speech recognition system. The underlying assumption behind most of these models is that the head will be viewed from only one known pose. As a result, these models are often only two-dimensional. Many are based directly on image features: Coianiz et al. [6] and Kass et al. [9] model the lips with contours along the outer edge, while Duchnowski et al. [7] feed the raw pixel intensities into a neural net to classify lip shapes. Others use such low level features to form a parametrization of the lip shape: Petajan et al. use several image features to estimate an overal lip contour [13]; Adjoudani et al. relate a small set of observed features (such as lip opening width and height, etc.) to the controls of a polygonal lip model [1].
Still others have a trained model of lip variations and attempt to fit the observations to this model. Some of the most interesting work done in this area has been along these lines: Bregler and Omohundro's work, for example [5], models the non-linear subspace of valid lip poses within the image space and can thus be used for both analysis and synthesis. Similarly, Luettin's system learns the subspace of variations for 2D contours surrounding the lips [11]. However, in order for these 2D models to be robust, they have to allow for at least small rotations of the head. The changes in the apparent lip shape due to rigid rotations, then, have to be modeled as changes in the actual lip pose. Our goal is thus to extend these ideas to $3 \mathrm{D}$. By modeling the true three-dimensional nature of the lips, variations that look complex and nonlinear from a $2 \mathrm{D}$ perspective become simple and linear.

With a $3 \mathrm{D}$ model, we can simply rotate the model to match the observed pose, thus modeling only the actual variations in lip pose. Some researchers have argued that only twodimensional information is observable and that it thus makes sense to base measurements and models on 2D features alone. However, while it is true that only the two-dimensional contours (at best) are visible in a given frame, the meaning of those two dimensions changes as the subject moves around - with changes is pose, the unobservables become observable. There has been some work done taking information from two known views [1], but this requires the head to remain fairly static. We feel that in order to capture interesting lip data during natural speech and gesture, it will be necessary to robustly track the lips from any pose. In addition, in order to fully train this model, it will be necessary to apply the observations from an arbitrary pose. Prior work has shown that the rigid position of the head can be robustly and accurately tracked [3], so it is feasible that we can apply the observations from any pose to the correct degrees of freedom of the model. As a result, our goal has been to create a model that can cover the full $3 \mathrm{D}$ variations of the lips.

The other category of lip models are those designed for synthesis and facial animation. These lip models are usually part of a larger facial animation system, and the lips themselves often have a limited repertoire of motions [10]. To their credit, these models are mostly in 3D. For many of the models, though, the control parameters are defined by hand. A few are based on the actual physics of the lips: they attempt to model the physical material and musculature in the mouth region [8],[15]. Unfortunately, the musculature of the lips is extremely complicated and has proved to be very difficult to model accurately. The basic physiology is comprised of an ellipsoidal muscle (the Obicularis oris) surrounding the mouth and several muscles which push and pull on this ring. This ellipsoidal muscle is exceedingly difficult to model in a computationally economic way. In addition, even if one were able to completely and correctly model the muscles and materials, the problem would not be solved: 
not have independent control of all of these facial muscles: the observed set of facial motions seem to be a slim subspace of the full range implied by the muscles. Some models, as in the work by Frisbie and Waters, have tried to approximate this subspace by modeling key lip positions (visemes) and then interpolating between them [15]. However, this limits the accuracy of the resulting lip shapes, since the only shapes learned from data are those for the static viseme poses.

We hope to fill the gap in these approaches with a 3D model that can be used for both analysis and synthesis. Our approach is to start with a $3 \mathrm{D}$ shape model and generic physics. We then deform this initial model with real $3 \mathrm{D}$ data to learn the correct physics and thus the correct modes of variation. We will demonstrate this technique using a simple finite element model and $3 \mathrm{D}$ point data, though the methodology could be applied to an arbitrary model and observation set. By training the modes of variation with real data, we can learn all of the deformation modes that occur in the observations. In this way, we not only solve the problem of parametrizing the model's motions, but also that of control. Because we learn only the modes that are observed, we end up with degrees of freedom that correspond only to plausible motions.

\section{The Model}

The underlying representation of our initial model is a mesh in the shape of the lips constructed from a linear elastic material. In the following section, we give a brief description of the choice of model shape and the physics used.

\subsection{The initial shape}

To get an initial shape for the model, a region surrounding the lips was extracted from a Viewpoint Data Labs model of the human head. There were some simple changes that were necessary to place this shape into a finite element framework. Because this mesh was designed for graphics and not for physics, there was some asymmetry between the right and left halves of the lips. By forcing these to be symmetric, it was ensured that the resulting stress-strain relations of the initial model would also be symmetric. In addition, some faces placed at strange angles for graphics effects were smoothed out to further facilitate the physical modeling. The final model has 336 faces and 204 nodes, resulting in 612 degrees of freedom (three per node). The initial shape of the model is shown in figure 1 .

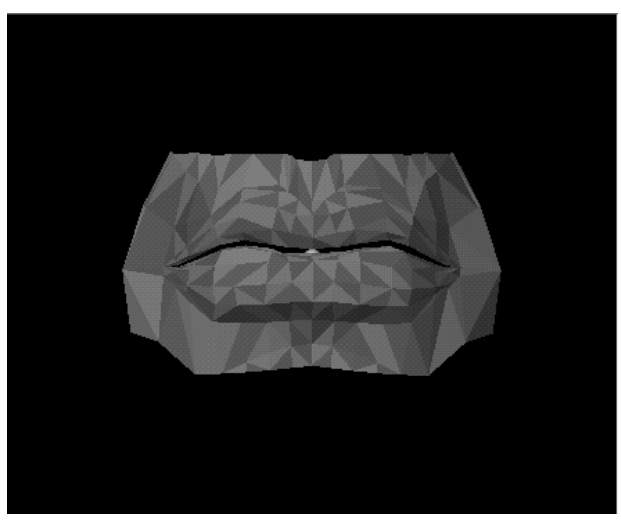

Figure 1: Initial shape of lips

\subsection{The finite element method}

The finite element method (FEM) is a numerical method for approximating the physics of an arbitrarily complex body. The central idea is to break the body into many small pieces whose individual physics are simple and then assembling the individual physics into a complete model. In contrast to the finite difference method, the finite element method also models the physics of the material between the nodes. This is possible because the method gives us interpolation functions that let us describe the entire modeled body in a piecewise analytic manner. Given this piecewise representation, the constitutive relations are integrated over the entire body to find the overall stress-strain relationship. The individual stress-strain matrices found in this manner can then be assembled into a single, overall matrix expressing the static equilibrium equation

$$
\mathrm{KU}=\mathbf{F}
$$

where the displacements $\mathbf{U}$ and forces $\mathbf{F}$ are in a global coordinate system. The details of this method are described in many references on finite elements including [4] and [16]. At the current time, we are not considering the higher order effects of dynamics (the mass and damping matrices). We hope to incorporate this into our model in the near future.

\subsection{Model Specifics}

For this application, a thin-shell model was chosen. We constructed the model by beginning with a $2 \mathrm{D}$ plane-stress isotropic material formulation [16] and adding a strain relationship for the out-of-plane components. For each triangular element, then, the six in-plane degrees of freedom are related with a six-by-six matrix $\mathbf{k}_{x y}$, while the out-of-plane degrees of freedom are related by the three-by-three $\mathrm{k}_{\mathrm{z}}$. In order to preserve the linearity of our model while maintaining the use of flat elements, we treat these two modes as being decoupled. They are thus assembled into the total $\mathrm{k}_{\mathbf{e}}$ as shown in block-matrix form below:

$$
\mathrm{k}_{\mathbf{e}}=\left[\begin{array}{ll}
\mathrm{k}_{\mathrm{xy}} & \\
& \mathrm{k}_{\mathrm{z}}
\end{array}\right]
$$

We built the 2D kxy using the formulation as described by Zienkiewicz [16] and Bathe [4]. This formulation has the following stress modes:

$$
\epsilon=\left[\begin{array}{c}
\epsilon_{x} \\
\epsilon_{y} \\
\gamma_{x y}
\end{array}\right]=\left[\begin{array}{c}
\frac{\partial u}{\partial x} \\
\frac{\partial v}{\partial y} \\
\frac{\partial u}{\partial y}+\frac{\partial v}{\partial x}
\end{array}\right]
$$

where $u$ and $v$ correspond to displacements along the $x$ and $y$ dimensions of the local coordinate system. Using the relation $\epsilon(\mathrm{x})=\mathbf{B}(\mathrm{x}) \mathbf{U}$ and the overall displacement vector

$$
\mathbf{U} \mathbf{e}=\left[\begin{array}{llllll}
u_{1} & v_{1} & u_{2} & v_{2} & u_{3} & v_{3}
\end{array}\right]^{T}
$$

we can solve for $\mathbf{B}$. In addition, the material matrix $\mathbf{C}$ is

$$
\mathrm{C}=\frac{E(1-v)}{(1+v)(1-2 v)}\left[\begin{array}{ccc}
1 & \frac{v}{1-v} & 0 \\
\frac{v}{1-v} & 1 & 0 \\
0 & 0 & \frac{1-2 v}{2(1-v)}
\end{array}\right]
$$

where $E$ is the elastic modulus and $v$ is Poisson's ratio. For the lip model, Poisson's ratio was chosen to be 0.01 . Since the elastic modulus $E$ is a constant multiplier of the entire material matrix, it can be used to vary the stiffness of the element is a whole. As a result, a default value of 1.0 was used for this 2 parameter. Elements that were to be more or less stiff than the 
default material were then assigned larger and smaller values respectively.

The next step is to relate the out-of-plane degrees of freedom. It is important at this stage to consider the desired behavior of the material. If it were important for nodes to be able to move independently out of the plane without causing strain in the adjoined nodes, the $\mathrm{k}_{\mathrm{z}}$ of equation 2 should be diagonal. In this case, however, it is desired that "pulling" on a given node has the effect of "pulling" its neighbors along with it. As a result, we construct the following $\mathrm{k}_{\mathrm{z}}$ :

$$
\mathrm{k}_{\mathrm{Z}}=\frac{E(1-v)}{(1+v)(1-2 v)}\left[\begin{array}{ccc}
1 & -0.5 & -0.5 \\
-0.5 & 1 & -0.5 \\
-0.5 & -0.5 & 1
\end{array}\right]
$$

Consider this matrix in terms of the basic relation keue $=f_{\mathbf{e}}$. A positive displacement (out of the plane) in only one of these degrees of freedom produces negative forces (into the plane) in the other two. This means that stretching one node out of the plane without moving the other two would require forces pushing the other two down. When equal displacements are applied to all three nodes, there is zero strain on the element, resulting in a rigid motion mode out of the plane. Though the out-of-plane degrees of freedom are decoupled from the in-plane components, this mechanism acts to relate the strain energy to the deformation of the element due to out-of-plane displacements. The greater the disparity in out-of-plane displacements (i.e., the greater the stretching of the element due to such motions), the greater the strain energy is produced by this $k_{z}$.

Once the degrees of freedom are properly aligned as described above, the resulting material has the approximate physics of many small plane-strain elements hooked together. The inplane forces of one element can pull on both the in-plane and out-of-plane components of its neighbors, and the vice versa. Once the complete $\mathbf{K}$ matrix has been assembled, we have a linear approximation to the relationship between the displacement and the resulting strain. We can now invert this relationship to find the displacements produced by an applied force (external strain). However, the matrix cannot be inverted as is: it is necessarily singular, as there are several displacement vectors that produce no stress in the body (i.e., they exist in the nullspace of $\mathbf{K})$. These are the modes of rigid motion. Consider, for example, a uniform displacement applied to all of the nodes. This would clearly produce no strain on the body. As a result, a minimum set of nodes must be "grounded" (i.e., held fixed) to prevent these singularities. For a $3 \mathrm{D}$ body, two nodes $(6$ DOF) must be grounded. This amounts to removing the rows and columns corresponding to the degrees of freedom for these nodes. The remaining $\mathbf{K}_{\mathbf{s}}$ has full rank and can be inverted to provide the desired strain-stress relation:

$$
\mathrm{K}_{\mathrm{s}}^{-1} \mathrm{~F}_{\mathbf{s}}=\mathrm{U}_{\mathbf{s}}
$$

while $\mathrm{K}$ is easy to compute and band diagonal (due to the limited interconnections between nodes), finding its inverse is an expensive calculation. We thus want to take this inverse only once at a point where it is appropriate to linearize the physics.

\subsection{Applying the method to the lips}

The method described above can be directly applied to the mesh in figure 1, resulting in a physical model in that shape made up of a uniform elastic material. However, in order to accentuate certain properties of the lips, some additional information was added to the model. First, in order to maintain smoothness in the inner contours of the lips, the faces along the inside ridges of the lips were made twice as stiff as the default material. In addition, to allow relatively free deformation of the lips while still maintaining the necessary rigid constraints, a thin strip of low-stiffness elements was added to the top of the lips stretching back into the oral cavity. The nodes at the far end of this strip were then fixed in 3D. Lastly, since the FEM linearizes the physics of a body around a given point, the initial K matrix was used to deform the model to the "rest state" of the lips (the method for this is described in a following section). The final $\mathrm{K}$ and $\mathrm{K}_{\mathrm{s}}^{-1}$ matrices were formed at this point, to allow for a greater effective range of the linearized physics. This $\mathrm{K}_{\mathrm{S}}^{-1}$ was then used for all remaining calculations. The final state of the model after adding these constraints and deformations can be seen in figure 7 .

\section{The Observations}

To train this lip model to have the correct $3 \mathrm{D}$ variations of the lips, it was necessary to have accurate 3D data. Also, in order to observe natural motions, it was not acceptable to affix reflective markers or other easily trackable objects to the lips. To satisfy these criteria, seventeen points were marked on the face with ink: sixteen on the lips themselves and one on the nose. The placement of these points is shown in figure 2. Note that only a subset of the points drawn on the lips (those that are numbered in figure 2) were used for tracking. The points were chosen to obtain a maximally informative sampling of the 3D motions of the lips. The spacing of the points accounts for the relative nonlinearity of various regions: regions where more non-linearity was expected were sampled more heavily. In addition, with the results of this study, it should become clear where the greatest remaining non-linearities reside, thus guiding the next stage of training.

It is important to realize that the choice of points is not inherently limited to those shown. As the development below will show, any set of observations can be used to train the model. Of course, the higher the information content of the data, the less data will be required to train the model. In addition, because the points would be tracked from two views in this case, it was necessary to choose points that were visible over a reasonable range of head rotation from both perspectives.

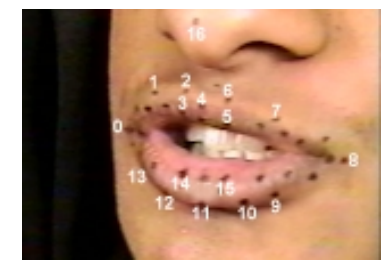

Figure 2: Locations of marked points on the face

Once the points were marked, two views of the points were taken by using a camera-mirror setup to ensure perfect synchronization between the two views (see figure 3 ). This resulted in two independent $2 \mathrm{D}$ views of the marked points. As can be seen in figure 5, the left side of the raw camera view contains the flipped version of the virtual (left) camera view.

The points were tracked over 150 frames at a $30 \mathrm{~Hz}$ frame rate using normalized correlation. Because of the large degree of motion of the lips between frames, normalized correlation did not always find the correct movement. As a result, a GUI was developed in which the user could move a tracker back to 


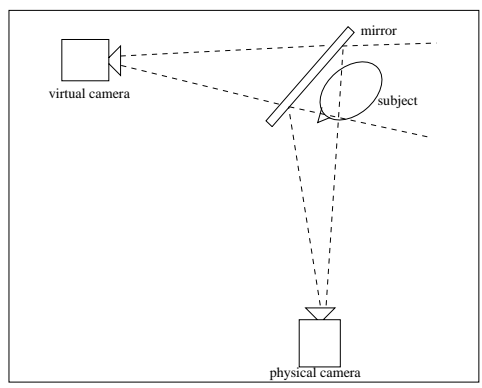

Figure 3: Data collection setup

It was attempted to have as great a variety of lip motions within this brief period as possible. To this end, several utterances using all of the English vowels and the major fricative positions were spoken during the tracking period. Clearly, 150 frames from one subject is still not enough to cover all possible lip motions, but it is enough to provide the model with the initial training necessary to cover a significant subset of motions. Methods to continue the training using other forms of input data will be discussed in a later section. The 3D camera calibration algorithm of Azarbayejani and Pentland [2] was then used to calibrate the real and virtual cameras using pinhole camera models. Given this calibration, the $3 \mathrm{D}$ point location for a given point was estimated by computing the closest point between the projective rays from the camera COP's (centers of projection) corresponding to that point. A schematic showing the projective rays for a sample 3D point are shown in figure 4. Also, a sample frame showing the $3 \mathrm{D}$ reconstructed points and the original video frame is shown in figure 5 . Note that the $3 \mathrm{D}$ points are shown at a different orientation than the camera views to demonstrate the reconstruction of depth information.

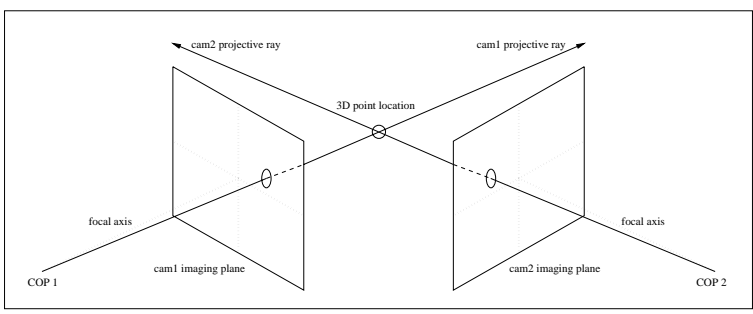

Figure 4: Point reconstruction geometry
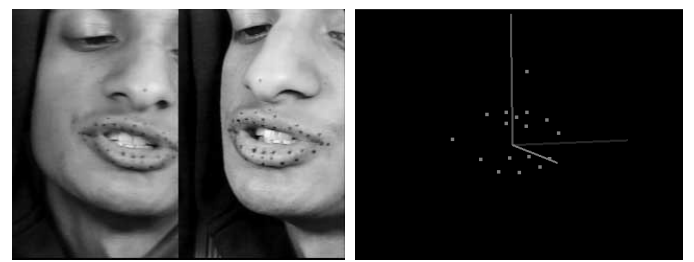

Figure 5: An input frame and the reconstructed 3D points

After the reconstruction, several steps were taken to normalize the input data. The position of the nose was subtracted out from all points to help account for rigid translation. In addition, to normalize the effects of rigid rotation, planes were fit to the outer subsets of both the upper and lower lip point sets.
The line forming the intersection of these planes was used to form the normalized coordinate: this line was treated as the $x$ axis, the $y$ axis was formed by taking the global $y$ and subtracting off the projection onto the new $x$, and the $z$ axis was the cross product between the two. An example of fitting planes to the points and finding the resulting coordinate system in shown in figure 6 below.

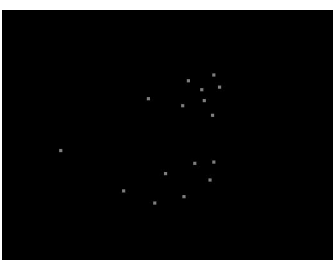

(a)

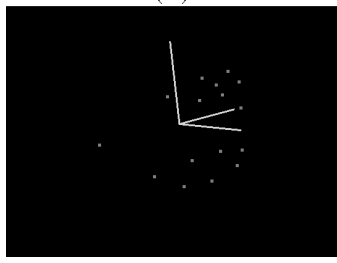

(c)

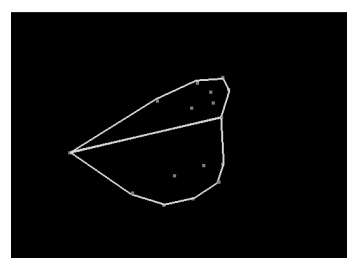

(b)

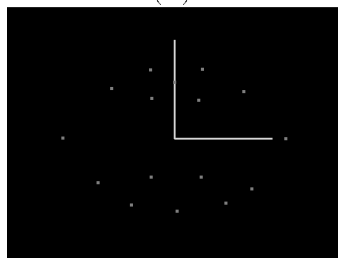

(d)
Figure 6: Normalization of 3D point coordinates

In order to then transform the training data into the coordinate frame of the model, a graphical interface was used to find the best rigid rotation, translation, and scaling that would fit the default model to the first frame of this data set. This transform was then inverted to bring the data into the normalized coordinate system of the model. The philosophy behind this is that all of the training should be in the coordinate system of the model; the resulting model can then easily be scaled and moved (in 3D) to fit new incoming data. This global normalization step was done only once for the entire data set.

\section{Training the Model}

In order to relate the training data to the model, the correspondence between data points and model nodes had to be defined. This was a simple process of examining a video frame containing the marked points and finding the nodes on the lip model that best matched them in a structural sense. The difference between the goal locations of these points (i.e., the observed point locations) and their current location in the model is then the desired displacement goal, $\mathbf{U}_{\mathbf{g}}$.

\subsection{Reaching the Displacement Goals}

The issue is now how to reach these displacement goals. The recorded data points have constrained 48 degrees of freedom (16 points on the lips with three degrees of freedom each). However, the other 564 degrees of freedom are left open. We thus have an underconstrained problem: there are an infinite number of ways to reach the desired displacement goals. However, we would not be satisfied with simply any one of these solutions - we want the one that minimizes the strain energy in the mesh. In other words, given the set of constrained point displacements, we want to find the set of displacements for the rest of the nodes that minimizes the strain felt throughout the structure. We denote the $\mathrm{K}_{\mathbf{S}}^{-1}$ matrix with all 600 columns but only the 
then describe the problem in the following LaGrange multiplier formulation: we wish to minimize

$$
\mathbf{F}^{\mathrm{T}} \mathbf{F}
$$

with the constraint

$$
\mathbf{P F}=\mathbf{U}_{\mathbf{g}}
$$

which results in the solution

$$
\hat{\mathbf{F}}=\mathbf{P}^{\mathbf{T}}\left(\mathbf{P P}^{\mathrm{T}}\right)^{-1} \mathrm{U}_{\mathrm{g}}
$$

This solution is thus a physically based smoothing operation: we are using the physics of the model to smooth out the regions where we have no observation data by minimizing the strain in the model.

A very interesting relationship exists between this solution and the linear least-squares estimation formulation. Consider treating the quantity we wish to estimate as the combined vector of $\mathrm{x}$ and $\mathrm{y}$ (i.e., $\mathrm{U}$ ), and the covariance matrix of the data as that found in equation 12. The LLSE estimate of $\mathbf{U}$ is then exactly the solution we obtained in equation 10 above. In other words, minimizing the strain in the model for a given set of displacement constraints is equivalent to finding the LLSE estimate of the displacements using the covariances resulting from the physical model. This can also be seen by realizing that the strain energy $\mathbf{F}^{\mathbf{T}} \mathbf{F}$ is the Mahalanobis distance using this covariance. Thus by minimizing the strain energy we are maximizing the likelihood of our solution given a Gaussian model of the distribution, which in the linear case (i.e., using only second order moments of the distribution) is equivalent to minimizing its mean-squared error regardless of the distribution. This provides a full-circle link between the covariance of the input data and the strain matrix of the model.

This method has thus given us displacements $\hat{\mathbf{U}}$ and forces $\hat{\mathbf{F}}$ for all 612 degrees of freedom for each frame. An example of using this method to deform the model is shown in figure 7 below. The deformed state shown was defined as the "rest state" of the lips as described above. The physics were thus re-linearized at this point, resulting in a final $\mathbf{K}_{\mathbf{S}}^{-1}$ that was used for the rest of the deformations.
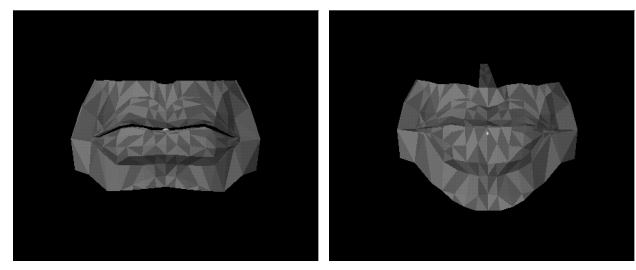

Figure 7: Initial model and deformation to rest state

Two frames of the original video along with the corresponding deformed model are shown in figure 8 below. Note that because the deformations of the model are shown in the coordinate system of the model, the deformations of the model will appear slightly different than the input data (applying the inverse of the rotation and independent scaling in $\mathrm{x}, \mathrm{y}$, and $\mathrm{z}$ that brought the data points to the model coordinate system would undo this effect).

\subsection{Modeling the Observations}

Once we have all the displacements for all of the frames, we can relate the observed deformations to a subset of the "correct" physics of the model. We began with the default physics
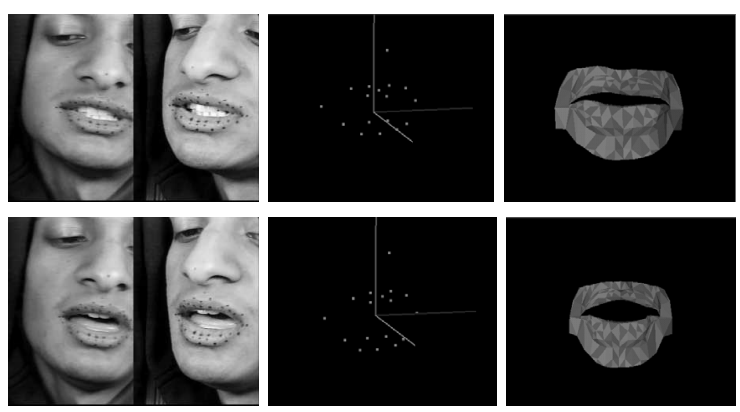

Figure 8: Example deformations of the model

(i.e., fairly uniform stiffness, only adjacent nodes connected) and have now observed how the model actually deforms. This new information can be used to form a new, "learned" $\mathbf{K}$ matrix. Martin et al. [12] described the connection between the strain matrix and the covariance of the displacements. Since $\mathrm{K}_{\mathrm{S}}^{-1}$ is a linear transform on $\mathbf{F}$, we can express the covariance of $\mathbf{U}$ in the following way:

$$
\mathbf{K}_{\mathbf{U}}=E\left[\left(\mathbf{K}_{\mathbf{s}}^{-1} \mathbf{F}\right)\left(\mathbf{K}_{\mathbf{s}}^{-1} \mathbf{F}\right)^{\mathbf{T}}\right]=\mathbf{K}_{\mathbf{s}}^{-1} E\left[\mathbf{F F}^{\mathbf{T}}\right]\left(\mathbf{K}_{\mathbf{s}}^{-1}\right)^{\mathbf{T}}
$$

If we now consider the components of the force to be IID with unit variance, the covariance matrix of $\mathbf{F}$ is the identity and we are left with

$$
\mathrm{K}_{\mathrm{U}}=\mathrm{K}_{\mathrm{s}}^{-1}\left(\mathrm{~K}_{\mathrm{s}}^{-1}\right)^{\mathrm{T}}=\mathrm{K}_{\mathrm{s}}^{-2}
$$

where the last step is possible because of the symmetry of $\mathbf{K}_{\mathbf{s}}$ and thus of $\mathbf{K}_{\mathbf{s}}^{-1}$. We can also take this mapping in the opposite direction: given the sample covariance matrix

$$
\hat{\mathbf{K}}_{\mathbf{U}}=E\left[(\mathbf{U}-\overline{\mathbf{U}})(\mathbf{U}-\overline{\mathbf{U}})^{\mathbf{T}}\right]=\frac{1}{n-1} \mathbf{A A}^{\mathbf{T}}
$$

where $\mathbf{A}$ is the matrix of observations in which each column is one observation $\mathbf{U}$ and $n$ is the number of observations. We can find $\mathrm{K}^{-1}$ by taking its square root, i.e., diagonalizing the matrix into $\mathbf{S} \boldsymbol{\Lambda} \mathbf{S}^{\mathbf{T}}$ (where each column of $\mathbf{S}$ is an eigenvector and $\Lambda$ is the diagonal matrix of eigenvalues) form and then reforming it with the square roots of the eigenvalues. We can then use the resulting "sample $K^{-1}$ " to represent the learned physics from the observations. Forces can now be applied to this matrix to calculate the most likely displacement given the observations.

However, because we only have a small number of observations (150) and a large number of degrees of freedom (612), we could at best observe 150 independent degrees of freedom. Furthermore, noise in the observations makes it unreasonable to estimate even this many modes. We thus take only the 10 observed modes that account for the greatest amount of variance in the input data. These modes are found by performing principal components analysis (PCA) on the sample covariance matrix [14], i.e., taking the eigenvectors and eigenvalues. Finding the eigenvalues and eigenvectors of the expected covariance matrix (which is 612 by 612) would take a great deal of computation. We can find the desired vectors by taking the eigenvectors of a much smaller matrix $\left(\frac{1}{n-1} \mathbf{A}^{\mathbf{T}} \mathbf{A}\right)$ and then appropriately transforming the results. Note also that because the matrices involved are symmetric, the eigenvectors will be orthogonal.

Once the eigenvectors were found, only the ten with the 5 largest eigenvalues were kept. These were then normalized to 
unit length and used to reassemble the sample covariance matrix as $\mathbf{S} \boldsymbol{\Lambda} \mathbf{S}^{\mathbf{T}}$. We can now use the sample covariance matrix to find the linear least squares estimate (LLSE) of the overall displacements given any subset of the displacements as an observation.

\section{Results}

The sample covariance was computed using only the first 140 frames so that the last ten could be used as a test set. After computing the covariance, the ten most expressive modes were found as described above. The mean displacement $(\overline{\mathbf{U}})$ and some of the first few modes are shown figure 9 below. It was found that the first ten modes cover 99.2 percent of the variance in the data - we should thus be able to reconstruct most shape variations from these modes alone.
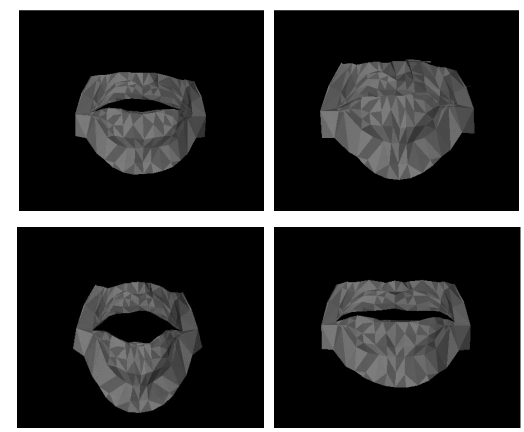

Figure 9: The mean displacement and some characteristic modes

To demonstrate how well this captures the variations in the data, the LLSE formulation was used to estimate the overall lip shape. As discussed earlier, one of the major arguments behind the $3 \mathrm{D}$ representation was that we could use any number of observations from any viewpoint and find the best estimate of the model shape. To demonstrate this capability, we have reconstructed the full shape using only $y-z$ data and only $x-y$ data (from all of the points) and $x-y-z$ data from only a subset of the points. For the ten data frames that were not included in the sample covariance, the mean-squared reconstruction errors per degree of freedom were found for several cases and are shown in the table below. The results are given in the coordinate system of the model, in which the model is 2.35 units across, 2.83 units wide, and 5.05 units deep. The first column of the table shows the reconstruction error using only the first ten modes; the second shows the error using the full covariance matrix. The fact that the performance is quite similar in both cases implies that the first ten modes have captured the important modes of variation in the data. The rows of the table correspond to what degrees of freedom were used to reconstruct the full 3D shape. In the first row, the first eight points (shown in figure 2) were used to reconstruct the remainder of the displacements. Note that the model performs quite well in this case, again implying that it has learned to some degree the permissible subset of lip motions. The second row shows the results of using only the $y$ and $z$ components of the data. This corresponds to the data that would be available from a profile view. The last row contains the results using the $\mathrm{x}$ and $\mathrm{y}$ components (i.e., a frontal view). It is interesting to note that the $y-z$ data provides much better peformance than the $x-y$ case. This is understandable in that there was a significant amount of depth variation in the test frames. Because some $x-y$ variations can occur with different degrees of $\mathrm{z}$ motion, the depth variation is not observable from the frontal plane. As a result, the $\mathrm{y}-\mathrm{z}$ data provides more information about the lip shape in these cases. Since our model is a full $3 \mathrm{D}$ representation, it can take advantage of this disparity (or any other advantageous 3D pose) when these observations are available.

Table 1: Reconstruction error per DOF (in normalized coordinates)

\begin{tabular}{|l|c|c|}
\hline Constraints Used & First 10 modes & Full Covar. \\
\hline \hline xyz (8 points) & $1.10 \mathrm{e}-3$ & $7.80 \mathrm{e}-4$ \\
\hline yz (all points) & $7.13 \mathrm{e}-4$ & $4.38 \mathrm{e}-4$ \\
\hline xy (all points) & $6.70 \mathrm{e}-3$ & $7.50 \mathrm{e}-3$ \\
\hline
\end{tabular}

\section{Conclusions and Future Directions}

With these preliminary experiments, the advantages of this full 3D analysis-synthesis model are clear. We can apply any number of observations for any points from any pose and robustly find the best lip shape estimate (although a minimum of points will be necessary for an accurate estimate). However, this is not the whole story. Clearly, it is not practical to mark and track points on every subject. To continue the training process, it will be necessary to use coarser features such as contours or color classification data. Though we will not have a direct correspondence with the model as in the case with point data, the relationship we presented between the covariance and the strain matrices allows us to use this information in a very different way. In essence, we can use the observed features to apply forces to the learned physics of the model. If a contour is not at the right position for the lip, it can apply a force in the right direction, and the learned physics will deform the rest of the model in an appropriate way.

\subsection{Improving the Model}

Because this model has only observed a subset of all possible lip motions, we cannot do the full deformation necessary for a new motion using only the learned physics. Clearly, as we have forced it to have a rank of only 10 , it cannot cover all possible variations of the lips. It is thus necessary to retain the original full-rank physical model in order to reach the new deformations. It is critical to use both the learned and the original models together. The learned model acts as a prior for the gross deformations and can quickly get us to the neighborhood of the correct displacement. The original model can then make small perturbations to this to make the final fit. Using the learned model alone cannot reach all of the deformations, and using the original model alone can produce improbable lip displacements since it has no notion of the range of possible lip motions. With this combined approach, we can to continue to train this model to cover all possible lip motions.

\section{$7 \quad$ Acknowledgements}

This work was partly supported by an NSF Graduate Research Fellowship. Also, a heartfelt thanks goes out to Irfan Essa for introducing me to FEM, his many helpful discussions, and for 6 all of his continued help and support. 


\section{References}

[1] A. Adjoudani and C. Benoit. "On the Integration of Auditory and Visual Parameters in an HMM-based ASR". In NATO Advanced Study Institute: Speechreading by Man and Machine, 1995.

[2] Ali Azarbayejani and Alex Pentland. Camera selfcalibration from one point correspondence. Technical Report 341, MIT Media Lab Vision and Modeling Group, 1995. submitted IEEE Symposium on Computer Vision.

[3] Sumit Basu, Irfan Essa, and Alex Pentland. "Motion Regularization for Model-Based Head Tracking". In Proceedings of 13th Int'l. Conf. on Pattern Recognition, August 1996.

[4] Klaus-Jurgen Bathe. Finite Element Procedures in Engineering Analysis. Prentice-Hall, 1982.

[5] Christoph Bregler and Stephen M. Omohundro. "Nonlinear Image Interpolation using Manifold Learning". In NIPS 7, 1995.

[6] Tarcisio Coianiz, Lorenzo Torresani, and Bruno Caprile. "2D Deformable Models for Visual Speech Analysis". In NATO Advanced Study Institute: Speechreading by Man and Machine, 1995.

[7] Paul Duchnowski, Uwe Meier, and Alex Waibel. "See Me, Hear Me: Integrating Automatic Speech Recognition and Lip-Reading”. In Int'l Conf. on Spoken Language Processing, 1994.

[8] Irfan A. Essa. "Analysis, Interpretation, and Synthesis of Facial Expressions". PhD thesis, MIT Department of Media Arts and Sciences, 1995.

[9] Michael Kass, Andrew Witkin, and Demetri Terzopoulous. "Snakes: Active Contour Models". International Journal of Computer Vision, pages 321-331, 1988.

[10] Y. Lee, D. Terzopoulos, and K. Waters. "Realistic Modeling for Facial Animation". In Proceedings of SIGGRAPH, pages 55-62, 1995.

[11] J. Luettin, N. Thacker, and S. Beet. "Visual Speech Recognition Using Active Shape Models And Hidden Markov Models". In ICASSP96, pages 817-820. IEEE Signal Processing Society, 1996.

[12] John Martin, Alex Pentland, and Ron Kikinis. Shape analysis of brain structures using physical and experimental modes. In CVPR94. IEEE Computer Society, 1994.

[13] E.D. Petajan. "Automatic Lipreading to Enhance Speech Recognition". In Proc. IEEE Communications Society Global Telecom. Conf., November 1984.

[14] Henry Stark and John W. Woods. Probability, Random Processes, and Estimation Theory for Engineers. Prentice Hall, 1994.

[15] K. Waters and J. Frisbie. "A Coordinated Muscle Model for Speech Animation". In Graphics Interface, pages 163$170,1995$.

[16] O.C. Zienkiewicz. The Finite Element Method in Structural and Continuum Mechanics. McGraw-Hill, 1967. 